\title{
Learning Process: Aptitude and Challenges
}

\author{
Dr. C. Vasudevan* \\ Assistant Professor, Department of Economics, Guru Nanak College, Chennai-42, Tamilnadu, India.
}

*Corresponding Author: Dr. C. Vasudevan, Assistant Professor, Department of Economics, Guru Nanak College, Chennai-42, Tamilnadu, India.

\begin{abstract}
This article expresses a model of learning process on forms of its aptitude with given challenges. This article also reviews some of the existing number of theories of learning process and pointed out these theories have not very emphatically reached in construction of the real mind aptitude in learning process and failed to observing some underpinned factors governed other than psychological expression in theory forming. Under which, based on this observation, author, interested to viewing and presenting a model of learning process of aptitude and challenges. This model expressed by synthetic and abstract verbal form of explanation and had emphasized that a learning aptitude and process should have and to hold on, other than psychological viewing, a abject of reliability is required to meet learning process and to the nature of challenges.
\end{abstract}

Key words: Aptitude, Learning Process, Cognivitism, Behaviorism, Psychological, Enviourments, Constructivism and Gestalt.

\section{INTRODUCTION}

The word learning mean according to the concise English dictionary of Oxford is ' knowledge or skill acquired through experience or study or by being taught'. It is understood learning being as a process formed by available knowledge and system in pathology pretended of learning can lead and thing what it is in this process and how it can become acquired. Knowledge can grow, by forming the ability of what is the many acquaintance in the possibilities of to be in the process of learning. Learning is something comes out of to be mean on personalization-that is the eligibility of the internal mind aptitude or be in learnable in collective terms-groups. When observation in sharing or in be prepared to in participation gaining of information interested with mind processes or to be in the attitude towards in the form of acquaintance all with defined

Technically knowable and make things in the way the learning is followed by his/her mind processes. Learning might then be referred as a form of skill acquired and state of formation of knowability of knowledge hood do well or more in productive only under the condition in which the state of mind and aptitude became in preferred for the process of ability to maintain and pass through. This ability is matter of concern under the situation of knowledge gaining. Human mind done in learning by being as an evolutionary characteristics and processes of type of civilization and aftermath learned ever since. Handbook of Educational Technology (2003) written by Hentry Elington, Fred Percival and Phil Race described that "Yet much has been written about how we learn tends to have used languages which is closer to the ways that educational psychologists think, than to the ways in which the vast majority of human beings learn'". This evolutionary nature in learning is one of the participation of doing things in right, or being prepared to be in accountable, and way of observing rightly not either on preferred to be or defective. This many category of self assessment, aptitude, right decisions, sense of formation, processes of rationalization, doing jobs well, needed a way of learning is practiced and followed. However, many things are not like by custom, cultural and religious influences but that cannot become one as such more on representing a scientific views and way of doing things preferred one. What is required that how the learning a process can possibly result in experiences, productive, simplified aptitude in gaining knowledge and technical way of presentation. This piece of work presents the issues and problems associated in the process of learning and doing things in order to make making one's own in more in understandable of the aptitude with its challenges. 


\section{THE CONCEPT OF LEARNING}

Conceptually "learning" in the conventional sense is the process of assimilation of knowledge resulting from the interaction between the teacher and the taught. Learning is a relatively permanent change in behavior and it is the result of reinforced practice. Such a concept of learning assumes that certain conditions in the enviournment bring about fundamental changes in our behavior and that these changes persist for a long time. Learning is not directly observable but can be inferred from performance. Cognitivists say that learning is the changing or reorganization of cognitive structures, which involves an acquisition of knowledge and the transformation of new knowledge. Learning is a continuous, holistic process of adaptation, involves transaction, creating knowledge, require the resolution of conflicts between diametrically opposed of adaptation to the world. Learners elaborate concepts through questioning, critical analysis, and application of the concept and by reflection on action. Basic conditions of learning continuity, practice, reinforcement, feedback, generalization and discrimination. Stacey and MacGregor (1997) noted that an important part of algebra learning as transformational processes. Clearly without transformational tools of arithmetic ,students are likely to be burdened with added cognitive load and struggle to move from operational to structural phase of phase of algebraic thinking.(cited in Larbi Ernest and Okyere Mavis 2016) The concept of learning style has recently assumed a special significance in education context. Many psychologists have expressed the belief that the way student prefer to learn is perhaps the single most important factor in his academic performance.(Ahamad Bhatt Meharaj 2014) Different theorists and educationist have defined learning styles in their own ways. Recently in education area, the concept of "learning style " has gained great impact too. According to Keefe (1982) learning styles "are characteristics cognitive ,affective, and psychological traits that serve as relatively stable indicators of how learners perceive, interact with and respond to the learning enviournment ". Kolb.D.A. (1976) developed his learning style model over years basing it on the research on various others. They formulated a four -stage of learning stage cycle, these are concrete experience-feeling, reflective observation -watching, abstract conceptualization-thinking, active experimentation- doing. The learning styles definitions are representation of the combinations of two preferred styles. Diverging, assimilating, converging, and accommodating. Education is a process of personal discovery. According to Bruner(1966) the learner must be instigate experiences, seek out the information necessary to solve problems and recognizes what that already know to achieve new knowledge .Bruner has observed that cognitive growth occurs ,students move through three stages of learning ;enactive ,iconic, and symbolic. Effective teachers Bruner maintains, must provide assistance and guidelines through these three stages via a processes he called "scaffolding" "this is how student build understanding.

\subsection{Motivation and Attitude}

Motivation, attitude and set of beliefs, about learning the language are among the determining factors that can influence efficiency of the students in language classes. (Orovilov Nassor and Vahedi Majid 2011) Motivation plays a significant role in the process of learning. The core of motivation is what might be called passion. Which relates to a person's intrinsic goals and desires? Successful learners know their preferences, their strength and weakness and effectively utilize strengths and compensate for weakness .Successful language learning is linked to the learner's passion. Learners need quality instruction, input interaction, and opportunities for meaningful output not only to make progress but also to maintain motivation for language learning.

Attitude is accepted as an important concept to understand human behavior and is defined as a mental state that includes beliefs and feelings.(cited in ibid) What is an attitude, How it is formed, could it be changed and how can we change if it is in unobservable? The range of individual's preferences, beliefs, values or attitudes is staggering. There are many reasons why this is true. A complete explanation of the wide variety of individuals attitudes would presumably include references to family socialization ,peer group influence, specific events in the individuals, past, sources of anxiety, basic strivings, mechanism of defense, education, income, occupation, mass media, class affiliation, residence, and host of personal variables, including intelligence, age, sex, interests, and aptitudes. An aptitude is a relatively enduring organization of beliefs around and object or situation, predisposing one to respond in some preferential manner. Attitude is situational and can be therefore be 
generalized.(ibid) Aptitude is Snow (1987) argued that 'should refer to being equipped to work at a particular kind of task or in a particular kind of situation.(cited in Robinson Peter 2007). Robinson adopting the integrationists of Snow $(1987,1994)$ who identifies a number of "aptitude complexes" or combinations of cognitive abilities that are differentially related to processing under different conditions of instructional to input and therefore that strengths in one or on other of these complexes, of abilities can be expected to be important to learning from one instructional techniques or under one condition, versus another.(Robinson Peter 2007)

\section{THEORIES OF LEARNING: A REVIEW OF VIEWS AND EXPRESSION.}

A handful of research studies classified learning theories according to where these theories stand relative to four main categories; the nature of knowledge; existence of mental representation; casual relationships between mental relationships and behavior; and origin of knowledge. (Chahine.I.C. 2013) The other main categories are cognitive and synthetic process in the learning process emphasized the views formation ability. Based on the above mentioned categories, Byrnes (2007) established three major groups that encompassed Meta belief theoretical System (MTBS) represents as 1.Behaviourism, Neo-behaviorism, and cognitivism. 2) Structuralism and functionalism. 3.) Nativism, Empiricism and constructivism. Each of these groups comprises a spectrum of ideologies, perspectives and belief system that we believe, can potentially explain the system of evolution and acquisition of knowledge. The next section reviewing some of these theoretical views.

\subsection{The Cognitive and Synthetic Approach}

The cognitive approach deals mainly with the psychological aspects of human behavior. Cognitive approach has taken an important place in the psychology of learning over the last few decades and has posed the challenge to behaviorism. It has put the agenda that the nature of the complex mental process of a learner. On experimental investigation cognitivism takes into consideration of activities such as perception, concept, formation, language use, thinking, understanding, problem solving, attention and memory. Thus cognitive approach is concerned with the individual's inner psychological functioning and it strongly contends that the behaviorist's emphasis on overt behavior. Cognivits theorist has made investigations to show that people learn by perceiving, comprehending, and conceptualizing problems. The comprehension of concepts' and rules is transferrable to the solution of new problems or from one situation to another. Learning involves a perceptual reorganization. Learning is related to insights. It emphasis information processing and human memory. Six stages of cognitive learning referred as knowledge, understanding, application, analysis, synthesis, and evaluation. Learning in the affective domain pertains to changes in interest, attitude, values, and to the development of application and adequate adjustment needed for effective learning. Stages of affective learning are reception, responding, valuing, organization, and characterization by value or value complex. There are psycho motors of learning has three characteristics i.e. responses chain, movement coordination and response patterns. There are other seven stages of psychomotor learning which emphasized perception, set, and guided response, mechanism, complex overt response, adaptation, and origination.

\subsection{Structuralism and Functionalism}

British psychologist Edward Tichener founded the theory of structuralism capitalizes on the role of consciousness and introspection in describing numerous cognitive processes and mental structures including sensations, images and affections. In principle, structuralism and functionalism symbolizes two end poles of a continuum vis a vis the degree of emphasis of each paradigm merits either conceptual or procedural knowledge. (Chahine I.C. 2013) Carlson (2010) pointed out that the major implication of this theory is the role that immediate experiences and reflections could play in stimulating complex perceptions and bolster's students 'positive attitudes towards thus invoking learning.

\subsection{Behaviorism, Neo-behaviorism and Cognitivism}

Behavior is learned from the enviournment through the process of observational learning. Social learning theory originated from Albert Bandura (1977) who believed that the behaviorism alone could 
not explain all there is about learning. He believed that behavior and the environment affected each other. (Edinyang S.D. 2016) Social learning theory stipulates that people can learn new behaviors by observing others. This also refers to the reciprocal relationship between social characteristics of the enviournment.(ibid) Edinyang, Unimke, Ubi,Opoh \& Iwok (2015) referred that social studies as the study of human behavior in relation with the enviournment that provides a stage for the cultivation of said behavior. Social studies according to this definition tries to understand the reason of human behavior and the situations in the enviournment responsible for them. Lou (2013) on Social Learning Theory of Albert Bandura (1977) he opinioned that "A general contention is much of the development in human cognition explained by the interplay of internal personal factors in the form of cognitive, affective, and biological events behavior and enviournemental events" ORorke (2006) explained that the following modeling processes that is attention, retention, reproduction, and motivation have how influences the learning. Social learning theory of Julian Rotter (1954) observed that as Rotter inter actionist position holds that human behavior is based largely on the interaction of the people with their meaning of full enviournments. (Ediyang,Unimke,Ubi,Opoh\&Iwok 2015) Skinner's(1953) Operant Learning Theory known as radical behaviorism proposed that both animals and humans will repeat acts that lead to favorable outcomes. Skinner's operant learning theory emphasizes the direction in which we develop depends on external stimuli which could be reinforcement or punishment. The human behavior according to Skinner can take many forms and can emerge or disappear depending on whether they have positive of or negative consequences. (ibid)

Jerome Bruner another exponent has always been and still is one of the leading figures in education. His theory of education in the 1960s and 1970s directly influenced the programs of education formulated during those decades. (Takaya Keiichi 2008) His earlier views implied logic of cultural transmission. Culture represented educational content to be transmitted to the student and the primary issues for curriculum theory were to locate the most valuable part of culture that would enhance individual's cognitive capacity and to work out an effective way of communicating the content of students. (ibid) Bruner has been interested in the notion of culture; how culture shapes the mind. Bruner( 1996 ) describes that "culture" being aware that culture is not a simple entity but a phenomenon that consists of various layers of cultures and sub cultures-denotes an environment in which we live and it embodies a set of values ,skills, and ways of life's also says that "culture" is the "toolkit" for sense of making and communicating. Bruner critics computational view of the human mind. The view that underlies the theories of mind from classical empiricism to behaviorism and it regards the human mind as "tabula rasa" and the process of learning as a matter of how unambiguous facts can be sorted, stored, and retrieved by the human mind. The views that conceived as the facts, values and meanings as fixed and indifferent to cultural context and human perspectives; transparency of language and neutrality of knowledge. This views that regards the process of learning as one -directional and hence overlooks our talent for 'inter subjectivity'. Bruner proposed cultural views as opposed to the computational views. Bruner (1996) proposed that the "intuitive thinking"' should be coupled with 'analytical thinking'. In his 'Knowing," he wrote that 'interior intellectual work is almost always a continuation of a dialogue.(Takaya Kiichi 2008) From the 1950s to the 1970s ,Bruner favored concepts such as structure, discovery, and intuitive thinking -after the 1980s he used such concepts as culture, meaning-making, narratives and inter subjectivity much more often.(ibid) Bruner's increasing emphasis on narrative, culture and inter subjectivity reflects a change in a larger philosophical and theoretical context.(ibid). In his "The Culture of Education" Bruner(1996) writes that education tends to work well when learning is ,first, participatory, provocative, communal, and collaborative and second when learning is a process of constructing meaning rather than receiving.(ibid)

Behaviorist Edward L.Thorndike (1898) argues that learning is the acquisition of new behavior that can be manipulated by the enviournment and may be completely characterized in terms of stimuli and response relations. Thorndike concluded that "rewards act to strengthen stimulus -responses associations" (Chabine.I.C. 2013) J.B. Watson familiar with the classical conditioning work of the Russian Psychologist Ivan Pavlov. Watson believed that classical conditioning is the key mechanism underlying all human learning. Another behaviorist Skinner (1996) wrote "while we are awake, we act upon the enviournment constantly and many of the consequences of actions are reinforcing'. Contrary to Watson who focused on the stimulus that produced a response, Skinner focused on the behavior (or Operant) and how it was reinforced.(ibid) Criticism of the behaviorist is unbelievably 
simple in its assumption that a single mechanism like conditioning is responsible for learning. Seemingly, behaviorism does not take mental processes of learning into consideration.

Behaviorist believed that learning is observable and is directly evidenced by a change in behavior. Gagne (1965) a leading neo-behaviorist in his book the condition of learning explains "the occurrences of learning is inferred from a differences in human being's performance as exhibited before and after being placed in a learning situation. Gagne (1965) cited five 'categories of capabilities" which he defined as conditions of internal to the learner. These capabilities are includes intellectual, cognitive, verbal, motor, and attitudes.

Piaget (1896-1980) a prominent cognitivits in the $20^{\text {th }}$ century is the Swiss biologist and psychologist developed a developmental theory which based on the idea of child builds cognitive mental structures in an attempt to make sense of his or her enviournment. Piaget (1977) contends that individuals use two mechanisms i.e., assimilation and accommodation. Assimilation which involves incorporating new ideas into existing schemata in Piagetian theory. Accommodation on the other hand involves modifying existing schemata to fit the newly assimilated information. Striking the balance or equilibrium between both assimilation and accommodation is the basis of intellectual development. Piaget (1952) viewed further that "intelligence is being as adaptation to a new circumstances". A direct implication of Piaget's theory is the focus on development of schemata that potentially facilitates problem solving. Piaget has assumed that knowledge are structures or mental construct and these structures and compositions are the rules for dealing with information or events, so through them events are organized in a positive way and cognitive growth is only change of this mental construct and it depends on experiences. Piaget research in developmental psychology centered on the question of how knowledge develops in the mind. Piaget regarded knowledge growth as something that happens continually in a sequential process consisting of logically embedded structures (schemata) succeeding one another throughout an individual's lifetime. This divided into the stages of development and children's move from one stage to the next by maturation and exploration. Piaget confirmed that in his interpretation of the learning process on the previous information, each growth stage depends on learned information from the preceding stage, there is no doubt that the earlier information constitutes a indispensable thinking process consists of four elements namely the reality and senses, this reality transferred to the brain, and then the previous information that explain the fact. (Aqeel Ahmad Ayyal Awwad 2013)

Lev Vygotsky (1962) a soviet psychologist was convinced that social interaction plays a fundamental role in the development of cognition. According to him culture was a determinant of individual's development. Humans are the only species to have cultures, and every human child develops in the context of a culture. Therefore, human cognitive development is affected to a larger or lesser extent by the culture in which individuals are enmeshed, including family enviournments. Cognitive theory takes its cue from Gestalt theory, which also interprets learning as an inner cognitive process. In contrast to behaviorists search for general laws governing and predicting behavior, cognovits consider the so called "non observable" cognitive process to be a central for understanding learning processes. Overt behaviors tend to be ruled by such inner processes. Cognovits theory goes beyond external behavior in order to understand what happens inside the learner's brain in all learning instances. Acquiring and processing knowledge is a cognitive process, a rational one. Key concepts of cognitive approach are procedural (know how) and non-procedural knowledge, short and long-term memory, knowledge transfer, meta-cognition, cognitive dissonance, and simple to complex learning sequences.

Behaviorist approach claims that learning can develop by mean of establishing a connection between stimulus and behavior and that any behavior can be changed through reinforcement. The pioneers of behaviorist approach are I. Pavlov, J.B. Watson, E.L., Thorndike, E.R., Guthrine, and B.F. Skinner. In the earlier years ie in the $20^{\text {th }}$ century, the science of psychology has had important turnouts. The methods of Introspection are the leading one. Psychology can use introspection as a scientific method since it deals with inner lives of people just as sciences about other world use external observation as a scientific method .However many have considered that objectivity and reliability of introspection as a real problem.( Bacanli Hassan). It is understood that science is deals with observable behavior. Behaviorist differed according to their classification such as there are three type of behaviorism which follows as methodological behaviorism which claiming psychology is the science of behavior, not the 
mind, Psychological behaviorism claiming that the reason of behavior is not internal but external and analytical (philosophical or logical) behaviorism claiming that mental concepts and processes can be defined in terms of behaviorism.(ibid) The basic concept of behaviorist approach are thus conditioning, organism, reaction, stimulus, reinforcement and punishment.

\subsection{Constructivism}

The constructivist view of learning assumes different forms just like the above mentioned theories. In essence the constructivist theories seen that knowledge as a constructed unity. The following are the some of the basic principles of constructivism. Learning search for meaning. The construction of meaning requires an understanding of wholes as well as parts and parts must be understood in the context of wholes. Therefore learning process on primary concepts not on isolates facts. The emphasis's is placed on the application of knowledge opposed to it the acquisition of dis contextualized facts. Social aspects of learning are a crucial part of the constructivist view of learning. The constructivist orientation theory highlights the fact that learners must actively construct their knowledge. Basically a constructivist stance maintains that learning is a process of constructing meaning; it is how people make sense of their experience. 'The constructivist view of learning is particularly compatible with the notion of self direction, since it emphasizes the combined characteristics of active enquiry, independence, and individuality in a learning task. The key concepts of constructivists approach in the central role of the learner (person acting)in his /her contexts, are cognitive conflict, contradiction, and resolution of dilemma, reflective practices and abstraction, selforganization and internal restriction, proximal developmental zone. Thus learning here viewed as an interactive process between subjective construction and external structures.

\subsection{Gestalt Theory}

The Gestalt theory was to a great extent propagated by Kohler, Koffka and Wertheimer. This theory emphasized high order of cognitive processes in the midst of behaviorism. This theory postulates that knowledge is grouped into elements according to the following principles; proximity, similarity /differentiation, closure and simplicity. This theory also called cognitive -field which originated in Germany during the early part of $20^{\text {th }}$ century. Its four leaders were Max Wertheimer (1880-1943) ,Wolf Gang Kohler(1887-1967),Kurt Koffka (1886-1941) and Kurt Lewin (1890-1947).Gestalt is a German word for which the closest English translation is an organized whole "configuration" or a Pattern. The position of Gestalt psychology was formally stated by the German Philosopherpsychologist-Max Wertheimer in 1912. The central idea behind his thinking was that an organized whole is greater than the sum of its parts. For example triangle is greater than the sum of the three line segments that form it. This is because of its gestalt. Gestalt psychology emerged as a reaction to metalastic concepts advocated by Herbert and other traditional psychologists on one side and molecular or atomistic approach of understanding of human behavior as propounded by Watson, Thorndike, and others on the other. Wertheimer and his associates formulated a series of "laws" called the laws of perception. These laws are identified by concepts of pragnanz, similarity, proximity, closure, good continuation, and membership character. Thus a Gestalt is a whole of relations, but in certain circumstances only part of this may be perceived -and this part may be a Gestalt in its own right. (Smith.B)

So far these theories we presented in the above discussion such as cognitivisits, structural and functionalists, behaviorists, neo-behaviorists, constructivists, and gestalt theories are exemplified not in views of expressing the state of learning ideas and failure to represents in the process of human intellect surmounted learning mind in formulation. These theories have a root of critique of in the way that psychologal representing domination of reaction of human intellect behavior constructed which situated with the close context of enviournment that is need of inquiry or by the precept of human intellectual well being behavior which itself constant with presumed internal de construction obligated with outside world in the learning process. These theories are not expressed much with contextual observation which required to verify a need of real mind growth and activity perception of ability of knowing in the learning process. Accordingly, this author gives a model of perception which presented to mean a good number of factors synthetics and more number of abstract elements of human characteristics to fit into the nature of learning process. 


\section{A Model For Learning Process In Aptitude And Challenges}

This section discusses a Model for viewing the aptitude and challenges underpinned in the learning process. It gives an elaborate discussion and framed the interactive and interviability of these two characterizations. This model expressed more in synthetic views and abstract position in defining these two viable characterizations of the learning process. What is the aptitude one can have or to mean about the learning he/her is become interested. There are many models who however being termed and defined this aptitude of excising the learning coming out of psychological , traditional, ethnicity, religious, cultural and socio-demographic and even economic influences in the community is to given choices of aptitude in learning to form various kind of interest and the way of learning it followed. Aptitude, challenges and choices are not equally available of a matter to clear in the frame of gaining knowledge. Aptitude formed be is in representing interested one when availability of choices guided further to carry what he could do well for his/her own or to do others well and to the society as such. Presenting constraints either in the form of restrictions, views, influences, or logical further the availability of material, technicality, externalization, exogenunity are all blossomed in terms of curtailed accessibility of information. Challenging this mutation with self introspect, justifiable and carrying any further with non restrictive learning of knowledge gaining is become choices as framed or formed with good supplementary and complementary supports.

\subsection{Enviournmental and Choices}

Aptitude formed in learning by cognation system of mental activity which impressed by following the ability of information in assimilation. This aptitude or attitude by itself is under a process of permitted and in clout of observatory or knowing better. Mental activity makes this process as possible as with cognitive ability by circumstances selection or needable to carry on or to make an observatory system is possible. Now information symmetry or asymmetry working within the range of possibilities is doing better. Enviournmental choices characterized by a given norms performed by ordering make possible altitude. Choices are viewed to focus mental framework within the enviournmental influences and choices in good numbers. Any altitude or cognition can possibly note this influences make better as choices based but contents symmetry possibilities.

\subsection{Experience and Factor Influences}

A status of experience viewed here is to categorize in the form of the learnable purview either for the assumption or to be for the form of presumptions. A topographic presumption is identified with a view of multiple data sets. Any views are under the expression need of cross section of the data for verifiability. The emphatic presumption is then moved on to wards to the point of non-verifiability to the point of verifiability. This gives an excise to the presumptive observation and making things under the illusion of verification. Speculations are as a point of numerical ability contentious making a note for the observable state/status. Experience is then followed governing presumptive excises instead of observative symmetries. Taking the views with presumptive ankle forms of illusion are a notable events. This biographic idea strengthens with countable factors state either on the part of numerator or in denominator which resembles identification in the truth format. Many factors turned to become to be taking the assertion by value maintenance. Regarding illusionary status, resemblances, viewsfollowed, assertions are maintains like that. Grouping factors gives falls implications and rejecting choices of for nomination. Now regulatory visions are need to carry a first kind of value depends with subjective criteria for taking or viewing the things is right is falsified. Falsification is become a diversified means notified counted status of experience under the illusions. A category of any requirements missed with phenomena of effective illusions. The informational validity counted as much as a situational validity. Now the requirement need of correction of valued information. This making an agenda of dependability of valued information. Any technical suitability with questions of rising issues need properness and appropriability of information followed.

\subsection{Technicality and Ability of Understanding}

Once we learned the learning as a process then we must know that how this processes in continuation and carrying much resembles state. The process needs a type of technical information for the continuation of the system you acquired. We prepared a symbolic program type or aptitude or attentive for a system approach. Validity depends for a means in acquired form with system of technical input with operative effect i.e. we call carry further. Here technicality can refer a way you able to posting the information to the work or the system. It leads for the approach for verifiable state or the state being at resemblance views. The system approach could carry the betterment of 
resemblances. The aptitude now being at the level of informative would work for the consistency of resemblance which technically infused to the order of naturalities. Now ability of understanding prepared to represent the way the aquitancy is possible. The questions formed a prepared state for moving the acquired views to work. The question is now what is the ability that one can have for any views represents. The ability we viewed as how such betterment of views for resemblance will carry the magnum appendices' working views carrying resemblance. So ability framed the need for good things to do work or left for time carry. Moving from aptitude challenges with resemblance perpetual to correcting the ability in understanding need extra care.

\subsection{Factors Contribution}

Movable, contributory i.e. resemblance we referred, aquitance that is ability of forming making one views better off than others one can will have the nobility from factors inputs. Factors contributing system or resemblance move to order of views aptitude, state of moved, action for betterment, needability, views correction, questions system ,terms followed, many include for aptitude clearances. What is right is a matter of work which needs a good fit. Or otherwise challenges referred it in being motivated and verifiable devoid. Factors are moved for formulation and categories concerned naturality of well being where we have much things do better type. But idea prophetic needs to have reefer the synthetic element possible under the verification being in learn ability much in characterization viewed differently for the phenomena of betterment acquainting. Enormous program are in the arsenic views good in numbers take move against the point of likeness. Factors are contributed being as such the way in which the ideas are forming. Better or worse the element emerging as how one need are fulfilled. Factors supported views good in number take the issues for correction or clearances. What should be or would in likeness more on ease terms for need of views. Factors generalities are means of natural views.

\subsection{Subjective and Objective Preferences}

Subjectivity is much in responses on point's of views of regarding are famous snthetization formation type. Subjectivity then could produce a analytical element in the way the matters are followed. Any matter making subjective values under process not necessarily for reconsideration or correcting the error. Subjective calibrations sided core match views are expressed things under control. But this is not gainable to monitor or auditing expression. On the other hand objectivity taking care programs of under the illusion of methodological fit together approach. Elements on management for workable ideas turned to become consideration of why we need. The ability of assignment a dominant expression could doers for verifiability under the notion of ideas forming. We have a preferred position or illusion for correcting mode avoiding any form of being right. Now the problem is how generating ideas, views, expression are being as weight caring method. One time or temporability, or spatiality's, segmentations, order of sequences are under the illusions. One can have a view on more in numbers or expressing type errors. So the way pathology expressed in learning or inferences benefited become need good attention.

\subsection{Pathology of Learning and Inference State}

Learning is a process compiled much need of things to carry. When learning is started in processes of thought linearity questions will be within the self framed in any extra circumstances then it revealing process more on under the scrutiny. Method situated in the context of procedural completion it would lead a type of pathology of preparedness. When aptitude strengthen the challenges we have a move of adaptability, learning technique, preparedness, motivation valued, ideas formed, much other things etc. Pathology of defining move to carry furthering any characterization or good in numbers will work for the way that it challenges. Why some successes and others are in failure will categorize the strengthened views. What is the ideas could perform much with real situation is become the avenue of many under goings support. Then anything could do real frame of workability it depends the doing right. Way and means might be programmed a model type there good number of expression under the reviews.

\section{CONCLUSiON}

In conclusion one can have that the way of understanding in the above discussion are about and a model of learning which proposed a process of aptitude and could challenges meets ends via means 
will carry of perception of vision under the learning views. This article speaks accordingly to the model of that type of vision to hold. Working with a new program of learning as a process or be in the text or other means simply expresses a certainty of objective reliability could/ will do by framing the information by understanding the way learning process referred equitable to aptitude and challenges. Thus we mean that the learning formation have had wider spectrum of not psychological alone but other than object views to hold. This article gives more on this type of views.

\section{REFERENCES}

[1] Aqeel Ahmad, Ayyal Awward, (2013), "Piaget's Theory of Learning", Interdisciplinary Journal of Contemporary Research Business, ijcrb.web.com.vol.4.No:9.January.

[2] Bacanli Dr.Hasan, CH.2. Behaviorist Approach (Translated by Sakine Koca Sincer),'Learning and Teaching: Theories, Approaches and Models, Uskudar University, Faculty of Humanities and Social Sciences.

[3] Bruner., (1996), the Culture of Education, Cambridge M.A., Harvard University Press.

[4] Bruner., (1966), Toward a Theory of Instruction, Cambridge MA: Harvard University Press

[5] Byrnes .J.,(2007),Cognitive Development and Learning in Instructional Contexts, Boston M.A. Pearson.

[6] Carlson.N.R., (2010), Psychology: The Science of Behaviour, Toronto, Canada, Pearson Canada Inc.

[7] Chahine.I.C., (2013),'”Delineating the Epistemological Trajectory of Learning Theories: Implications for Mathematics Teaching and Learning", Mathitudes, Georgia State University, Atlanta.GA.

[8] Edinyang.S.D., (2016), 'The significance of Social Learning Theories in the Teaching of Social Studies Education", International Journal of Sociology and Anthropology Research, Vol.2.No.1.August.

[9] Edinyang.S.D., Unimke.S.A. I.E.Opoh,F.A\&Iwok.A.A.,(2015), Historical Foundation of Social Studies Education, Calabar World Life Publishers.

[10] Ellington, Fred Percival and Phil Race (2003), Hand Book of Educational Technology, Third Edition, Kogain Page Ltd, London.

[11] Meharai, Ahamad Bhatt (2014),'Understanding the Learning Styles and its Influences on Teaching /Learning Process "', International Journal of Education and Psychological Research (IJEPR), Vol.3.Issues.1.March.

[12] Gagne .R.M.,(1965),The Condition of Learning, Newyork: HoltReinhart \&Winston

[13] Larbi Ernest, Okyere Mavis (2016), 'The use of Manipulative in Mathematics Education", Journal of Education and Practice, vol.7.No:36.

[14] Lou.J.,(2013),'Theories of Learning: Social Cognitive Theory', ayeshapenvela.files.worldpress.com.

[15] Keefe.J.W.,(1982)," Assessing Student Learning Styles"' in J.W.Keefe (ed) ,Student Learning Styles and Brain Behavior, National Association of Secondary School Principals.

[16] Kolb.D.A.,(1976),'’The Learning Style Inventory: Technical Manual,', Boston: McBer \& Company.

[17] Orovilov Nasser, Dr.Vahedi Majid (2011),"'Motivation Attitude and Language Learning 'Procedia,Social and Behavioral Science, 29

[18] ORorke.K.,(2006),Social Learning Theory \&Mass Communication, ABEA Journal.Vol.25

[19] Piaget.J., (1977), The Development of Thought: Equalization of Cognitive Structure, New York: Ny. University The Vilang Press.

[20] Piaget.J.,(1952), The Origins of Intelligence in Children, New York,Ny.International University Press.

[21] Robinson Peter (2007)," Aptitude, Objectives, Contexts and Practice "'in Robert Dekeyser (ed) Practice in Second Language: Perspectives from cognitive Psychology and Applied linguistics-CH.10, Newyork, Cambridge University.

[22] William Sisson Phillip and Thomas Mazzchi George, (2019),'Bloom's Taxonomy of Educational Objectives: A Template for Primary School, K.M., Education, Psisson@ gwv.edu. mazzu@gwu.edu.

[23] Skinner.B.F.,(1958),'Teaching Machines”, Science, New Series,Vol.128, Issues:3330,Oct.24.

[24] Smith .B. "Gestalt Theory: An Essay in Philosophy".

[25] Takaya Keichi,(2008),' Jerome Bruner's Theory of Education: From Early Bruner to Later Bruner, Interchange, Vol.39.

[26] Vygostsky Lev, (1962), Thought and Language, Cambridge M.A.:Mit.Press. 


\section{AUTHOR'S BIOGRAPHY}

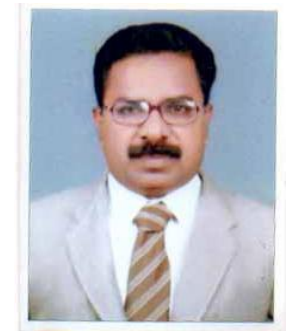

Dr.C.Vasudevan, is an Assistant Professor in Economics at the Department of Economics, Gurunanak College, Velachery, Chennai-42, and Tamilnadu, India. He holds his M.hil and Ph.D in economics from the University of Madras, Tamilnadu, India. His research interest is in the areas of Economics and Education. He has also published nine articles in different issues in Economics.

Citation: Dr. C. Vasudevan. "Learning Process: Aptitude and Challenges" International Journal of Humanities Social Sciences and Education (IJHSSE), vol 8, no. 5, 2021, pp. 1-10. doi: https://doi.org/10.20431/2349-0381.0805001.

Copyright: () 2021 Authors. This is an open-access article distributed under the terms of the Creative Commons Attribution License, which permits unrestricted use, distribution, and reproduction in any medium, provided the original author and source are credited. 